\title{
Promiscuous esterases counterintuitively are less flexible than specific ones
}

\author{
Christina Nutschel ${ }^{1}$, Cristina Coscolín ${ }^{2}$, Daniel Mulnaes ${ }^{3}$, Benoit David ${ }^{3}$, Manuel Ferrer ${ }^{2}$, \\ Karl-Erich Jaeger ${ }^{4,5}$, Holger Gohlke (1,3,* $^{\text {, }}$ \\ ${ }^{1}$ John von Neumann Institute for Computing (NIC), Jülich Supercomputing Centre (JSC) and \\ Institute of Biological Information Processing (IBI-7: Structural Biochemistry), \\ Forschungszentrum Jülich GmbH, 52425 Jülich, Germany \\ ${ }^{2}$ Institute of Catalysis, Consejo Superior de Investigaciones Científicas, 28049 Madrid, Spain \\ ${ }^{3}$ Institute for Pharmaceutical and Medicinal Chemistry, Heinrich Heine University Düsseldorf, \\ 40225 Düsseldorf, Germany \\ ${ }^{4}$ Institute of Molecular Enzyme Technology, Heinrich Heine University Düsseldorf, 52425 \\ Jülich, Germany \\ ${ }^{5}$ Institute of Bio- and Geosciences IBG-1: Biotechnology, Forschungszentrum Jülich GmbH, \\ 52425 Jülich, Germany
}

Running title: Flexibility and promiscuity of esterases

Keywords: Esterase promiscuity, structural flexibility, thermostability, Constraint Network Analysis, TopModel, TopScore, conformational proofreading

*Corresponding author:

John von Neumann Institute for Computing (NIC), Jülich Supercomputing Centre (JSC), and Institute of Biological Information Processing (IBI-7: Structural Biochemistry)

Forschungszentrum Jülich GmbH

Wilhelm-Johnen-Straße

52425 Jülich

Germany

Email: gohlke@uni-duesseldorf.de or h.gohlke@,fz-juelich.de 


\section{Abstract}

35 Understanding mechanisms of promiscuity is increasingly important from a fundamental and application point of view. As to enzyme structural dynamics, more promiscuous enzymes generally have been recognized to also be more flexible. However, examples for the opposite received much less attention. Here, we exploit comprehensive experimental information on the substrate promiscuity of 147 esterases tested against 96 esters together with computationally

40 efficient rigidity analyses to understand the molecular origin of the observed promiscuity range.

41 Unexpectedly, our data reveal that promiscuous esterases are significantly less flexible than 42 specific ones, are significantly more thermostable, and have a significantly increased specific activity. These results may be reconciled with a model according to which structural flexibility in the case of specific esterases serves for conformational proofreading. Our results signify that esterase sequence space can be screened by rigidity analyses for promiscuous esterases as starting points for further exploration in biotechnology and synthetic chemistry. 


\section{Introduction}

Enzymes involved in primary metabolism typically exquisitely discriminate against other metabolites. Yet, evolution of specificity is only pushed by nature to the point at which 'unauthorized' reactions do not impair the fitness of the organism (1). As a result, the universe of promiscuous activities available in nature has been suggested to be enormous $(2,3)$. Understanding mechanisms of promiscuity has thus become increasingly important for the fundamental understanding of molecular recognition and how enzyme function has evolved over time(4) but also to optimize enzyme engineering applications (5). A particular challenge in the latter case is the ability to discover a suitable enzyme with 'sufficient' promiscuous activity to serve as a starting point for further exploration (1).

Enzyme structural dynamics, besides its role in catalysis $(6,7)$ and allosteric regulation $(8-11)$, has been recognized as likely the single most important mechanism by which promiscuity can be achieved (5). Prominent examples are human cytochrome P450 (CYP) enzymes, for which crystallographic studies and molecular simulations demonstrated that more promiscuous CYPs show larger structural plasticity and mobility (12-14), or TEM-1 $\beta$-lactamase and a resurrected progenitor, for which molecular simulations show that the pocket of the ancestral, and more promiscuous, enzyme fluctuates to a greater extent (15). However, examples for the opposite, i.e., conformational changes selected in evolution such that they enhance specificity in molecular recognition (16), have received much less attention in the context of enzyme promiscuity.

A clear limitation for scrutinizing the link between enzyme structural dynamics and substrate promiscuity is the general lack of large-scale data on one enzyme (super)family tested against a multitude of ligands (17) (cf. ref. (1) for notable exceptions). Likewise, acquiring information on enzyme dynamics at the atomistic level by experimental techniques or classical molecular dynamics (MD) simulations is burdensome. Here, we exploit comprehensive experimental information on the substrate promiscuity (18) of esterases (abbreviated EHs, for "Ester Hydrolases") (19) together with computationally efficient rigidity analyses (20-23) of comparative models of EHs to understand the molecular origin of the observed promiscuity range. Enzyme rigidity, or its opposite flexibility, are static properties that denote the impossibility, or possibility, of motions in an enzyme under force, without giving information about directions and magnitudes of movements (23). Enzyme flexibility, thus, should not be confused with enzyme mobility, which describes actual motions in an enzyme. Rigidity analysis results do not rely on the correct description of the time-dependency of processes (23), which 
makes them valuable in cases where timescales over multiple orders of magnitude may govern such processes, like in enzyme dynamics $(6,7)$.

In recent years, EHs have obtained much attention in basic research and industrial applications (24). EHs are widely distributed in nature within microbial communities (at least one EH is found in each bacterial genome), they have been extensively examined with state-of-the-art (meta)genomics techniques and investigated by functional screenings compared to many other classes of enzymes. They also possess outstanding properties in terms of stability, reactivity, and scalability that make them appropriate biocatalysts to improve competitiveness, innovation capacity, and sustainability in a modern circular bio-economy (25). Recently, a large-scale study on substrate promiscuity ( $P_{\mathrm{EH}}$, which denotes the number of esters hydrolyzed by an $\left.\mathrm{EH}\right)$ of 147 phylogenetically, environmentally, and structurally diverse microbial EHs was described by Martínez-Martínez et al. (19), in which all EHs were functionally assessed against a customized library of 96 esters. As to mechanistic understanding, the authors related $P_{\mathrm{EH}}$ to a structural parameter, the active site effective volume. However, the impact of enzyme flexibility on $P_{\text {EH }}$ was not assessed.

In our study, we thus ask the following questions: I) What is the relation between $P_{\mathrm{EH}}$ and EH flexibility? II) Does this relation hold if experimentally determined EH thermostabilities are used as proxies for enzyme flexibility? III) What is the relation between $P_{\mathrm{EH}}$ and EHs' specific activities? IV) Is there a preference of promiscuous or specific EHs for a particular type of esters. V) Can this preference be understood with respect to EHs flexibilities? 


\section{Materials and Methods}

\subsection{Definition of data sets}

The present study builds on the study from Martínez-Martínez et al. (19). In order to assess $P_{\mathrm{EH}}$, the authors experimentally investigated 147 phylogenetically, environmentally, and structurally diverse microbial EHs (termed experimental data set) against a customized library of 96 different esters. Two commercial lipases, which have found wide biotechnological applications, CalA and CalB from Pseudozyma aphidis (formerly Candida antarctica), were included for comparison. For details on determining and classifying $P_{\mathrm{EH}}$, see Supplemental Materials and Methods. To validate that $P_{\mathrm{EH}}$ defines promiscuity of EHs in a quantitative manner, $k_{\text {cat }}$ and $K_{\mathrm{m}}$ values were determined for ten expressed and purified EHs covering the entire $P_{\mathrm{EH}}$ range (see section 2.9) and a promiscuity index $I$ (see Supplemental Materials and Methods, Eq. S4) computed and compared to $P_{\mathrm{EH}}$. Finally, the similarity of the ester substrates was assessed by the maximum pairwise Tanimoto-Combo similarity score $\delta_{i j}$ for compound $i$ versus $j$, accounting for shape and chemical complementary between $3 \mathrm{D}$ structures, and the mean maximum pairwise Tanimoto-Combo similarity score $\delta_{i}$ of a substrate $i$ to all other substrates in the data set (see Supplemental Methods and Materials).

As our computational approach involves extensive molecular dynamics (MD) simulations for generating conformational ensembles (see section 2.3), we selected 35 EHs from the volume data set (termed flexibility data set) for comparative modeling (see section 2.2). The criteria for choosing EHs of the flexibility data set are explained in section 3.1.

\subsection{Comparative modelling and validations of the flexibility data set}

Comparative models of the flexibility data set (see section 2.1) were generated using our inhouse structure prediction meta-tool TopModel (26) that has been successfully applied in previous studies (27-30). TopModel uses multiple state-of-the-art threading and sequence/structure alignment tools to generate a large ensemble of models from different pairwise and multiple alignments of the top five highest ranked template structures. The TopModel software is available at https://cpclab.uni-duesseldorf.de/index.php/Software.

The quality of the homology models was assessed by our meta Model Quality Assessment Program (meta-MQAP) TopScore (31). TopScore uses deep neural networks (DNN) to 
combine scores from 15 different primary MQAP to predict accurate residue-wise and wholeprotein error estimates. For details on model quality assessment by TopScore and validation, see Supplemental Materials and Methods.

To test whether CARs of the homology models are accessible for substrates, we applied the CAVER 3.0.3 PyMOL Plugin (32). Starting points for the computations were defined based on the Cartesian coordinates of the CARs' center of mass (COM). Default values were used for the probe radius $(0.9 \AA)$, shell radius $(3.0 \AA)$, and shell depth $(4.0 \AA)$.

\subsection{Generation of structural ensembles}

Structural ensembles of EHs were generated by all-atom MD simulations of in total $5 \mu \mathrm{s}$ simulation time per EH. For details on starting structure preparation, parametrization, and equilibration see Supplemental Materials and Methods.

All minimization, equilibration, and production simulations were performed with the pmemd.cuda module (33) of Amber19 (34). During production simulations, we set the time step for the integration of Newton's equation of motion to 4 fs following the hydrogen mass repartitioning strategy (35). Coordinates were stored into a trajectory file every 200 ps. This resulted in 5000 configurations for each production run that were considered for subsequent analyses.

\subsection{Constraint Network Analysis}

The flexibility analyses were performed with the Constraint Network Analysis (CNA) software package (version 3.0) (20-23). CNA functions as front- and back-end to the graph theory-based software Floppy Inclusions and Rigid Substructure Topography (FIRST) (36). Applying CNA to biomolecules aims at identifying their composition of rigid clusters and flexible regions, which can aid in the understanding of biomolecular structure, stability, and function (21-23). As the mechanical heterogeneity of biomolecular structures is intimately linked to their diverse biological functions, biomolecules generally show a hierarchy of rigidity and flexibility (20). In CNA, biomolecules are modeled as constraint networks in a body-and-bar representation, which has been described in detail by Hesphenheide et al. (37). A fast combinatorial algorithm, the pebble game, counts the bond rotational degrees of freedom and floppy modes (internal, independent degrees of freedom) in the constraint network (38). In order to monitor the 
163 hierarchy of rigidity and flexibility of biomolecules, CNA performs thermal unfolding simulations by consecutively removing non-covalent constraints (hydrogen bonds, including salt bridges) from a network in increasing order of their strength (39-41). For details on thermal unfolding simulations, see Supplemental Materials and Methods. To improve the robustness and investigate the statistical uncertainty, we carried out CNA on ensembles of network topologies $\left(\mathrm{ENT}^{\mathrm{MD}}\right)$ generated from MD trajectories (see section 2.3) (42).

The CNA software is available under academic license at https://cpclab.uniduesseldorf.de/index.php/Software and the CNA web server is accessible at https://cpclab.uniduesseldorf.de/cna.

\subsection{Local and global indices}

From the thermal unfolding simulations, CNA computes a comprehensive set of indices to quantify biologically relevant characteristics of the protein's stability. Global indices are used for determining the rigidity and flexibility at a macroscopic level; local indices determine the rigidity and flexibility at a microscopic level of bonds (43). The cluster configuration entropy $H_{\text {type2 }}$ is a global index that has been introduced by Radestock and Gohlke (20). As done previously, we applied $H_{\text {type2 }}$ as a measure for global structural stability of proteins $(20,41,44-$ 48). The stability map $r c_{i j}$ is a local index that has been introduced by Radestock and Gohlke (20). We applied $r c_{i j}$ as a measure for local structural stability of proteins in previous studies $(45,47,48)$. For details on both indices, see Supplemental Materials and Methods.

\subsection{Root mean square fluctuations}

The per-residue root-mean-square fluctuations were calculated for each $\mathrm{EH}\left(R M S F_{\mathrm{EH}}\right)$ and for its CARS ( $R M S F_{\mathrm{CAR}}$ ) based on the MD trajectories (see section 2.3). Prior to the calculations, the structures of each trajectory were superimposed onto the average structure using the $90 \%$ least mobile residues of the respective EHs (49). 


\subsection{Torsion angles}

For each of the 96 esters, the number of freely rotatable bonds (torsion angles, TA) was calculated based on the SMILES codes provided by Martínez-Martínez et al. (19).

To compare how many esters with a specific TA are hydrolyzed by each $\mathrm{EH}$, we calculated the normalized proportion of ester hydrolysis with a specific TA (Normester(TA)) as the number of hydrolyzed esters with a specific TA (Esterhydrolysed (TA)) divided by the total number of esters in the data set with this specific TA (Ester library(TA)) (Eq. 1).

$$
\operatorname{Norm}_{\text {ester }}(T A)[\%]=\frac{\text { Ester }_{\text {hydrolyzed }}(T A)}{\text { Ester }_{\text {library }}(T A)} * 100 \%
$$

\subsection{Circular dichroism spectroscopy}

Eleven serine ester hydrolases, EH1 (Protein data Bank acc. nr. 5JD4), EH2 (GenBank acc. nr. KY483643), EH3 (GenBank acc. nr. KY483645), EH4 (GenBank acc. nr. KR107250), EH6 (GenBank acc. nr. KP347751), EH8 (GenBank acc. nr. WP_011587341.1), EH9 (GenBank acc. nr. KY483648), EH16 (GenBank acc. nr. KP347759), EH21 (GenBank acc. nr. KP347760), EH37 (GenBank acc. nr. KR107248), EH43 (GenBank acc. nr. KP347758) from metagenomic origin, were used in the present study to perform CD determinations. The vector pET46 Ek/LIC and the host Escherichia coli MC1061 were the source of the His6-tag EH $\mathrm{EH}_{1}, \mathrm{EH}_{4}, \mathrm{EH}_{8}$, and $\mathrm{EH}_{37}$, the vector pBXNH3 and the host E. coli MC1061 were the source of the His6-tag $\mathrm{EH}_{2}, \mathrm{EH}_{3}$, and $\mathrm{EH}_{9}$, and the vector p15Tv-L and the host E. coli BL21(DE3) were the source of $\mathrm{EH}_{6}, \mathrm{EH}_{16}$, $\mathrm{EH}_{21}$, and $\mathrm{EH}_{43}$. Prior to analyses, the soluble His-tagged proteins were produced and purified after binding to a Ni-NTA His-Bind resin (Sigma-Aldrich, MO, US) as described by MartínezMartínez (19). Purity was assessed as $>98 \%$ using SDS-PAGE analysis in a Mini PROTEAN electrophoresis system (Bio-Rad, Madrid, Spain) and subsequent staining with Coomassie Brilliant Blue. A total of about 10-20 mg total purified recombinant protein was obtained on average from 1-liter culture. All proteins were stored at $-20^{\circ} \mathrm{C}$ at a concentration of $10 \mathrm{mg} / \mathrm{ml}$ in $40 \mathrm{mM}$ (4-(2-hydroxyethyl)-1-piperazineethanesulfonic acid (HEPES) buffer (pH 7.0), until used. Circular dichroism (CD) spectra were acquired between 190 and $270 \mathrm{~nm}$ with a Jasco J720 spectropolarimeter equipped with a Peltier temperature controller, employing a $0.1 \mathrm{~mm}$ cell at $25^{\circ} \mathrm{C}$. Spectra were analyzed, and denaturation temperatures were determined at $220 \mathrm{~nm}$ between 10 and $85^{\circ} \mathrm{C}$ at a rate of $30^{\circ} \mathrm{C}$ per hour, in $40 \mathrm{mM}$ (4-(2-hydroxyethyl)-1- 
piperazineethanesulfonic acid (HEPES) buffer, $\mathrm{pH}$ 7.0. A protein concentration of $1.0 \mathrm{mg} \mathrm{ml}^{-1}$ was used. Denaturation temperatures were calculated by fitting the ellipticity (mdeg) at $220 \mathrm{~nm}$ at each of the different temperatures using a 5-parameters sigmoid fit with Sigma Plot 14.0.

\subsection{Kinetic parameter determination}

Ten serine ester hydrolases, EH1 (Protein data Bank acc. nr. 5JD4), EH3 (GenBank acc. nr. KY483645), EH5 (GenBank acc. nr. KR107271), EH7 (GenBank acc. nr. KY483644), EH12 (GenBank acc. nr. KR107263), EH17 (GenBank acc. nr. KR107278), EH37 (GenBank acc. nr. KR107248), EH102 (Protein data Bank acc. nr. 5JD3), EH114 (GenBank acc. nr. KR107274) and $\mathrm{EH}_{127}$ (GenBank acc. nr. KR107253) from metagenomic origin, were used in the present study to perform kinetic determinations $\left(k_{\mathrm{cat}}\right.$ and $K_{\mathrm{m}}$ ). The vector pET46 Ek/LIC and the host Escherichia coli MC1061 were the source of the His6-tag EH1, EH5, EH12, EH17, EH37, EH102, $\mathrm{EH}_{114}$, and $\mathrm{EH}_{127}$, and the vector $\mathrm{pBXNH3}$ and the host $E$. coli MC1061 was the source of the His6-tag $\mathrm{EH}_{3}$. The soluble His-tagged proteins was produced and purified as described by Martínez-Martínez (19). For details see above.

The kinetic parameters were calculated at $550 \mathrm{~nm}$ using a continuous $\mathrm{pH}$ indicator (Phenol red; $\varepsilon 550 \mathrm{~nm}=8450 \mathrm{M}^{-1} \mathrm{~cm}^{-1}$ ) assay at $550 \mathrm{~nm}$ in 384-well plates as previously described (Alonso et al, 2020). Briefly, to $40 \mu \mathrm{l}$ of $5 \mathrm{mM}$ 4-(2-hydroxyethyl)-1-piperazinepropanesulfonic acid (EPPS) buffer ( $\mathrm{pH}$ 8.0) $2 \mu \mathrm{l}$ of a stock ester solution was added to achieve the desired concentration of each ester. Finally, $2 \mu 1$ of stock protein solution was immediately added to each well, to achieve the desired protein concentration, using an Eppendorf Repeater M4 pipette (Eppendorf, Hamburg, Germany). The total reaction volume was $44 \mu 1$. Ester hydrolysis was measured at $30^{\circ} \mathrm{C}$ in a Synergy HT Multi-Mode Microplate Reader in continuous mode at 550 $\mathrm{nm}$ over $24 \mathrm{~h}$. One unit (U) of enzyme activity was defined as the amount of free enzyme or enzyme bound to the carrier required to transform $1 \mu \mathrm{mol}$ of substrate in $1 \mathrm{~min}$ under the assay conditions using the reported extinction coefficient (Phenol red at $550 \mathrm{~nm}=8450 \mathrm{M}^{-1} \mathrm{~cm}^{-1}$ ). For $K_{m}$ determination - [protein]: $4.5 \mu \mathrm{g} \mathrm{ml}^{-1}$; [ester]: 0-100 $\mathrm{mM}$; reaction volume: $44 \mu \mathrm{l}$; $\mathrm{T}$ : $30^{\circ} \mathrm{C}$; $\mathrm{pH}$ : 8.0. For $k_{\text {cat }}$ determination - [protein]: 0-270 $\mu \mathrm{g} \mathrm{ml}^{-1}$; [ester]: $100 \mathrm{mM}$; reaction volume: $44 \mu \mathrm{l}$; $\mathrm{T}: 30^{\circ} \mathrm{C}$; $\mathrm{pH}$ : 8.0. All values, in triplicates, were corrected for non-enzymatic transformation. Kinetic parameters were calculated by fitting the data fit with Sigma Plot 14.0. 


\section{Results}

\subsection{Definition of data sets}

To understand the structural origin of and develop a method to predict $P_{\mathrm{EH}}$, the present study builds on large-scale data from Martínez-Martínez et al. (19). The authors experimentally investigated $P_{\mathrm{EH}}$ of $147 \mathrm{EHs}$ (termed experimental data set) (see section 2.1). In doing so, compromises needed to be made regarding the measurement of catalytic activity, i.e., specific activity was measured using enzymes expressed in E. coli without subsequent purification, only a single concentration of wet cells expressing enzymes ( $0.4 \mathrm{mg}$ per ester) was used to measure activity, and the substrates were tested at a single concentration of circa $7 \mathrm{mM}$ on average (19). To validate that the $P_{\mathrm{EH}}$ derived from the measured activities define promiscuity of the enzymes in a quantitative manner, $k_{\mathrm{cat}}$ and $K_{\mathrm{m}}$ values were determined now for ten expressed and purified EH covering the entire $P_{\mathrm{EH}}$ range (see section 2.9). $k_{\mathrm{cat}} / K_{\mathrm{m}}$ ideally serves as the kinetic parameter in enzyme promiscuity studies for comparison $(50,51)$. From $k_{\text {cat }} / K_{\mathrm{m}}$ values of an enzyme towards a defined set of substrates, a quantitative index of promiscuity $I$ (Eq. S4) can be calculated based on information entropy (51). I yields a very good and significant $\left(R^{2}=0.79\right.$, $p=0.0003$ ) correlation with $P_{\mathrm{EH}}$, indicating that $P_{\mathrm{EH}}$ relates to $\mathrm{EH}$ promiscuity in a quantitative manner (Figure S1), although the range of $I$ suggests that large $P_{\mathrm{EH}}$ may still be associated with moderate promiscuity. Note that, although the promiscuity index is a functional parameter that is defined for a specified set of substrates, promiscuity indices for different enzymes are quantitatively comparable if they have been calculated using the same substrate set (51). Furthermore, for the ten $\mathrm{EH}$ and using the colorimetric assay herein used, $k_{\mathrm{cat}} / K_{\mathrm{m}}$ can be measured with a standard error of the mean as low as $0.05 \mathrm{~min}^{-1} \mathrm{mM}^{-1}$, which corresponds to $k_{\text {cat }}$ and $K_{\mathrm{m}}$ fitting values 2 -fold above the background signals under assay conditions for each of the enzymes and esters. When $k_{\mathrm{cat}} / K_{\mathrm{m}}>0.05 \mathrm{~min}^{-1} \mathrm{mM}^{-1}$ is used as a criterion to define that a substrate is hydrolyzed, the resulting number of substrates for the ten EHs yields an excellent and significant $\left(R^{2}=0.99, p<10^{-4}\right)$ correlation with $P_{\mathrm{EH}}$ (Figure S2), again indicating that $P_{\mathrm{EH}}$ relates to EH promiscuity in a quantitative manner. Finally, esters that are chemically similar to each other are expected to be metabolized similarly by an EH; such correlations in the substrate set would reduce the effective EH promiscuity. Therefore, similarity of the substrates was assessed by the maximum pairwise Tanimoto-Combo similarity score $\delta_{i j}$ for compound $i$ versus $j$, which is bounded between 0 for dissimilar compounds and 2 for identical ones, and the mean maximum pairwise Tanimoto-Combo similarity score $\delta_{i}$ of a substrate $i$ to all other substrates in the data set; the Tanimoto-Combo similarity score accounts for shape and chemical 
complementary between 3D structures as determined by the Rapid Overlay of Chemical Structures approach (52). Complete linkage clustering on the pairwise distance matrix calculated for all 96 compounds from $\delta_{i j}$ yielded 20 clusters at a distance of 1.0 (Figure S3), which is equivalent to $\delta_{i j}=1.0$, indicating that on average less than five esters share a similarity that is half-way between dissimilar and identical. The negatively skewed histogram of $\delta$ i furthermore shows that $\delta$ peaks at 1.0 and is below 1.2 (Figure S4), indicating that an ester generally shares a similarity to all other esters that is half-way between dissimilar and identical, or worse.

Additionally, Martínez-Martínez et al. ranked (classified) $P_{\mathrm{EH}}$ of 96 EHs (termed volume data set) based on the active site effective volume (see section 2.1) (Eq. S1) (19), which will be used here as a reference to compare the power of $P_{\mathrm{EH}}$ predictions. As our computational approach involves extensive MD simulations for generating conformational ensembles (see section 2.3), we selected $35 \mathrm{EHs}$ from the volume data set based on the following criteria; they constitute the flexibility data set. I) The data set contains eleven EHs with known crystal structures (including the commercial EHs CalA and CalB) (Figure 1A, Table S1) and 24 EHs for which no experimental structure is available but for which comparative models can be generated (see section 3.2) (Figure 1A, Table S2). That way, we can probe to what extent the source of structural information influences the outcome of our results. II) The chosen EHs of the data set show high diversities as to $P_{\mathrm{EH}}$ and association to esterase families $\left(F_{\mathrm{EH}}\right.$, as defined by Arpigny and Jaeger (53)), similar to those found for the volume data set (Figures S1 and S2, Tables S3 and S4). This resulted in $P_{\mathrm{EH}}$ ranging from 4 to 72 (Figure 1A, Tables S1 and S2). In the following, we consider $P_{\mathrm{EH}}$ as low if the EH hydrolyzes $\leq 9$ esters (11\% of the data set), as moderate if the $\mathrm{EH}$ hydrolyzes between 10 and 29 esters (49\%), and as high if the $\mathrm{EH}$ hydrolyzes $\geq 30$ esters (40\%) (Figure S5, Table S3). The data set covers eleven FEH of which FIV (44\% of the data set) and Fv (21\%) are the best represented ones (Figure S6, Table S4). This reflects the proportion of their presence in the volume data set. III) Only EHs with amino acid sequence identities $\geq 25 \%$ in comparison to an existing crystal structure were considered (see section 2.1) in order to ensure a sufficient quality of generated comparative models.

Finally, in order to uniformly depict the results across the present study, six EHs were selected as representatives of the flexibility data set based on $P_{\mathrm{EH}}$ (termed representative data set): EHs with the lowest (EH115) or highest $P_{\mathrm{EH}}(\mathrm{EH} 001)$ and known crystal structures, EHs with the lowest (EH127) or the highest $P_{\mathrm{EH}}$ (EH005) and unknown crystal structures, and commercial EHs with the lowest (CalA) or highest $P_{\mathrm{EH}}(\mathrm{CalB})$ (Figure 1A-D, Tables S1 and S2). 


\subsection{Comparative models of EHs generated by TopModel show an overall and residue-} wise good quality

320

321

322

323

324

325

To generate structural models of EHs as starting points for our investigations, we performed template-based modeling of the flexibility data set using TopModel (26) (see section 2.2). In doing so, we also generated comparative models of the eleven EHs for which crystal structures are available. These structural models will be used to judge the quality of the comparative modeling.

The quality of the comparative models of the flexibility data set were assessed with TopScore (31), a meta Model Quality Assessment Program (meta-MQAP) (see section 2.2). For the eleven Es with known crystal structure, the global TopScores range from 0.074 to 0.305 (Figure 1A, Table S1). As the global TopScore describes whole-protein error estimates, this shows that the structures contain between 7.4 and $30.5 \%$ error. Notably, the global TopScores well and significantly correlate $\left(R^{2}=0.61, p=0.004\right)$ with values of 1 - IDDT computed from comparisons of the comparative models of EHs with known crystal structure against these experimental reference structures, indicating that global TopScores are well suited to assess the model quality of EHs (Figure S7, Table S5). The global TopScore values of the comparative models of the other $24 \mathrm{EHs}$ range from 0.087 to 0.269 (Figure 1A, Table S2), indicating that these models are of equal quality than the ones for EHs with known crystal structure. The TopScore values of the representative data set lie in a comparable range (Figure 1A, Tables S1 and S2). Moreover, the comparative models of the flexibility data set show low residuewise TopScore values (31), indicating that all parts of a model are of good quality. We illustrate this for the residue-wise TopScore of the comparative models of the representative data set (Figures 1B-D). This also applies to structural regions around CARs (Figures 1B-D). That way, it was possible to confirm CARs in models of EHs with known crystal structures and to unambiguously identify CARs in models of EHs with unknown crystal structures (Figures 1BD, Tables S1 and S2).

Additionally, we validated that CARs in all models are accessible for substrates according to CAVER results (32) (see section 2.2), i.e., that all models are in an open conformation: CARs are either located on the protein surface or are buried and connected with the surface by tunnels. We illustrate this for the comparative models of the representative data set (Figure S8). 
To conclude, comparative models were generated for $35 \mathrm{EHs}$ of the flexibility data set using TopModel. The models showed both an overall and residue-wise good structural quality. Additionally, we validated that CARs in all models are accessible for substrates.

\subsection{Promiscuous EHs are globally less flexible}

Previous studies indicated that enzyme flexibility influences the substrate promiscuity of enzymes (12-14). For gaining insights into how the flexibility of EHs is linked to $P_{\mathrm{EH}}$, we applied CNA $(21,23)$, a rigidity theory-based approach to analyze biomolecular statics (21-23), to the flexibility data set (see sections 2.4). To improve the robustness and investigate the statistical uncertainty, for each of the comparative models we carried out CNA on ensembles of network topologies (ENT ${ }^{\mathrm{MD}}$ ) generated from five MD trajectories of $1 \mu \mathrm{s}$ length each (44) (see sections 2.3 and 2.4). In order to investigate if the global flexibility of the EHs influences $P_{\mathrm{EH}}$, we predicted $T_{\mathrm{p}}$, the phase transition temperature previously applied as a measure of structural stability of a protein $(20,41,44-48)$, for each $E H$ (see section 2.5). $T_{\mathrm{p}}$ was averaged over five ensembles (see sections 2.3 and 2.4), resulting in all but one case in SEM $<1.87 \mathrm{~K}$ (Figure 2A, Tables S1 and S2).

$T_{\mathrm{p}}$ and $P_{\mathrm{EH}}$ of the flexibility data set are well and significantly correlated $\left(R^{2}=0.60, p=5.4^{*} 10^{-}\right.$ ${ }^{8}$ ) (Figure 2A). To validate the consistency of our approach, we considered EHs with known or unknown crystal structures separately. In both cases, good and significant correlations between $T_{\mathrm{p}}$ and $P_{\mathrm{EH}}$ were revealed (known crystal structures: $R^{2}=0.48, p=0.019$; unknown crystal structures: $R^{2}=0.73, p=1.1^{*} 10^{-7}$ ), lending support to the quality of comparative models predicted with TopModel and indicating that future predictions on EHs with unknown experimental structures should be promising. Notably, EHs with high $P_{\mathrm{EH}}$ have a high $T_{\mathrm{p}}$ and vice versa, i.e., promiscuous EHs are globally less flexible. Exemplarily, this is depicted for EHs of the representative dataset with known crystal structures and lowest (EH115) or highest $P_{\mathrm{EH}}(\mathrm{EH} 001)$, which showed $T_{\mathrm{p}}$ of $322.3 \mathrm{~K}$ and $357.2 \mathrm{~K}$, with unknown crystal structures and lowest (EH127) or highest $P_{\mathrm{EH}}(\mathrm{EH} 005)$, which showed $T_{\mathrm{p}}$ of $318.6 \mathrm{~K}$ and $351.1 \mathrm{~K}$, and CalA and CalB, which showed $T_{\mathrm{p}}$ of $346.2 \mathrm{~K}$ and $351.6 \mathrm{~K}$ (Figure 2A, Tables S1 and S2). The differences in global structural stability of these EHs are illustrated by the rigid cluster decomposition at $332 \mathrm{~K}$ during the thermal unfolding simulations (Figure 2B-D): promiscuous EHs are globally more structurally stable at the elevated temperature as indicated by fewer, but larger, rigid clusters. 
The EH flexibility analyzed so far is a static property and describes the potential of motions in a biomolecule (23). Yet, direct information on mobility within EHs is available from the ensembles generated by MD simulations. We thus computed exemplarily $R M S F_{\mathrm{EH}}$, a measure for protein mobility (see section 2.6), across the ensembles of EHs from the representative data set. $R M S F_{\mathrm{EH}}$, averaged over all residues and all five MD trajectories, and $P_{\mathrm{EH}}$ do not yield a significant correlation ( $p=0.13$ ) (Figure S9A, Table S6), in contrast to $T_{\mathrm{p}}$ and $P_{\mathrm{EH}}\left(R^{2}=0.93\right.$, $p=1.8^{*} 10^{-3}$ ) (Figure S9B, Table S6). Still, as promiscuous EHs are globally less mobile, the same trend is obtained as in the case of the flexibility analysis.

To conclude, a good and significant correlation between $T_{\mathrm{p}}$ and $P_{\mathrm{EH}}$ was found for the flexibility data set $\left(R^{2}=0.60, p=5.4^{*} 10^{-8}\right)$. These findings demonstrate that promiscuous EHs are globally less flexible. $R M S F_{\mathrm{EH}}$ is less predictive for $P_{\mathrm{EH}}$, although again promiscuous $\mathrm{EHs}$ are characterized by a lower global mobility, mutually confirming either result.

\subsection{Promiscuous EHs are more thermostable}

Previous studies indicated that thermodynamically more thermostable proteins frequently have a higher structural stability $(45,48)$. In order to investigate if promiscuous EHs, which were predicted to be less flexible (see section 3.3), are also more thermostable, CD spectroscopy was applied to determine the melting temperature $T_{\mathrm{d}}$ of the EHs (see section 2.8). Note that only if the unfolding of a protein is reversible, $\mathrm{CD}$ spectroscopy provides true thermodynamic properties (54). However, even if the unfolding is irreversible, because the protein aggregates at high temperatures, the method can still give information about relative stabilities (54). Hence, to reduce the potential impact of different aggregation kinetics of structurally different proteins, we applied CD spectroscopy to one $F_{\mathrm{EH}}$ family only. In particular, we used $F_{\mathrm{IV}}$ because it is the largest $F_{\mathrm{EH}}$ (Table S7).

Exemplarily, a CD spectrum for $T_{\mathrm{d}}$ determination is shown for EH001 (Figure 3A); for each $\mathrm{EH}, T_{\mathrm{d}}$ determination was performed in triplicates with $\mathrm{STD}<0.62 \mathrm{~K} . T_{\mathrm{d}}$ and $P_{\mathrm{EH}}$ yield a fair and significant correlation $\left(R^{2}=0.32, p=0.033\right.$, Figure 3B; a similarly fair and significant correlation is found if the data point with highest $T_{\mathrm{d}}$ is omitted $\left.\left(R^{2}=0.45, p=0.016\right)\right)$.

To conclude, promiscuous EHs are not only globally less flexible but also more thermostable. 


\subsection{Promiscuous EHs have less flexible catalytically active residues}

The good correlation of $P_{\mathrm{EH}}$ and $T_{\mathrm{p}}$ encouraged us to investigate if local flexibility characteristics of CARs will provide an even better predictor of EH promiscuity. We thus computed Flex $\mathrm{CAR}$ for the flexibility data set, i.e., the stability of rigid contacts between CARs and other residues that are at most $5 \AA$ apart from each other, based on the local index $r c_{i j, n e i g h b o r}$ (see section 2.5). For each EH, Flex $\mathrm{CAR}_{\mathrm{R}}$ was averaged over five ensembles (see sections 2.3 and 2.4), resulting in SEM $<0.06 \mathrm{kcal} \mathrm{mol}^{-1}$ (Figure S10A, Tables 1 and 2).

Flex $\mathrm{CAR}_{\mathrm{C}}$ and $P_{\mathrm{EH}}$ of the flexibility data set yield a good and significant correlation $\left(R^{2}=0.51, p\right.$ $\left.=1.7 * 10^{-6}\right)$ (Figure S10A). To validate again the consistency of our approach, we considered EHs with known and unknown crystal structures separately. In both cases, good and significant correlations between Flex $\mathrm{CAR}_{\mathrm{C}}$ and $P_{\mathrm{EH}}$ were found (known crystal structures: $R^{2}=0.63, p=$ $3.7^{*} 10^{-3}$; unknown crystal structures: $R^{2}=0.47, p=2.2 * 10^{-4}$ ), again lending support to the quality of comparative models predicted with TopModel. Hence, EHs with high $P_{\mathrm{EH}}$ have low FlexCAR and vice versa, i.e., promiscuous EHs have less flexible CARs. Exemplarily, this is detailed for EHs of the representative dataset with known crystal structures and lowest (EH115) or highest $P_{\mathrm{EH}}$ (EH001), which showed Flex $\mathrm{CAR}$ of $-0.74 \mathrm{kcal} \mathrm{mol}^{-1}$ and $-1.91 \mathrm{kcal} \mathrm{mol}^{-1}$, with unknown crystal structures and lowest (EH127) or highest $P_{\mathrm{EH}}(\mathrm{EH} 005)$, which showed FlexCAR of $-1.10 \mathrm{kcal} \mathrm{mol}^{-1}$ and $-1.86 \mathrm{kcal} \mathrm{mol}^{-1}$, and CalA and CalB, which showed Flex $\mathrm{CAR}$ of -1.31 $\mathrm{kcal} \mathrm{mol}^{-1}$ and $-1.95 \mathrm{kcal} \mathrm{mol}^{-1}$ (Figure S10A, Tables S1 and S2). The differences in local structural stability of these EHs are illustrated by rigid contacts between CARs and other residues that are at most $5 \AA$ apart from each other (Figure S10B-D): promiscuous EHs are locally more structurally stable as indicated by more stable rigid contacts.

Finally, we exemplarily computed $R M S F_{\mathrm{CAR}}$, a measure for the mobility of a protein's CARs (see section 2.6), across the ensembles of EHs from the representative data set. Averaged $R M S F_{\mathrm{CAR}}$ and $P_{\mathrm{EH}}$ correlate worse $\left(R^{2}=0.74, p=0.029\right)$ (Figure S11A, Table S6) than Flex $\mathrm{CAR}$ and $P_{\mathrm{EH}}\left(R^{2}=0.92, p=2.4 * 10^{-3}\right)$ (Figure S11B, Table S6), paralleling the above results for the global measures. Still, again, as promiscuous EHs have less mobile CARs, the same trend is obtained as in the case of the flexibility analysis.

To conclude, a good and significant correlation between Flex $x_{\mathrm{CAR}}$ and $P_{\mathrm{EH}}$ was found for the flexibility data set $\left(R^{2}=0.51, p=1.7^{*} 10^{-6}\right)$. Hence, promiscuous EHs have less flexible CARs. $R M S F_{\mathrm{CAR}}$ is less predictive for $P_{\mathrm{EH}}$, although again promiscuous EHs are characterized by less mobile CARs, mutually confirming either result. 


\subsection{Promiscuous EHs have an increased specific activity}

444

445

446

447

448

449

450

451

452

453

454

455

456

457

458

459

460

461

462

463

464

465

466

467

468

469

470

471

In the study by Martínez-Martínez et al. (19), the experimental data set was screened against 96 esters in a kinetic $\mathrm{pH}$ indicator assay (see section 2.1). Besides the average specific activity Act $t_{\text {average }}$ given in $\mathrm{U} /$ ( $\mathrm{g}$ wet cells), also the average maximum specific activity Act $_{\max }$ was determined. Motivated by the reactivity-selectivity principle (RSP) initially introduced for organic chemistry reactions (55), which states that a more reactive chemical compound is less selective in chemical reactions, we intended to probe if $P_{\mathrm{EH}}$ is related to Actmax. For this, we established an approximate linear free-energy relationship (LFER) (56) by relating $\log \left(A c t_{\max }\right)$ and $\log \left(P_{\mathrm{EH}}\right)$ (Figure S12, Table S8). In this analysis, the CalA and CalB preparations were excluded because $A c t_{\max }$ was given in $\mathrm{U} /$ ( $\mathrm{g}$ total protein) there.

$\log \left(\right.$ Act $\left._{\max }\right)$ and $\log \left(\mathrm{P}_{\mathrm{EH}}\right)$ of the experimental data set yield a good and significant correlation $\left(R^{2}=0.50, p=4.6^{*} 10^{-23}\right)$ (Figure S12A). Likewise, $\log \left(\operatorname{Act} t_{\max }\right)$ and $\log \left(P_{\mathrm{EH}}\right)$ of the flexibility data set yield a fair and significant correlation $\left(R^{2}=0.22, p=0.6^{*} 10^{-2}\right)$ (Figure S12B). To validate whether the same trend emerges for EHs with known and unknown crystal structures, we considered both types of EHs separately. In both cases, fair and significant correlations between $\log \left(A c t_{\max }\right)$ and $\log \left(P_{\mathrm{EH}}\right)$ were found (known crystal structures: $R^{2}=0.34, p=0.099$; unknown crystal structures: $R^{2}=0.23, p=0.019$ ).

To conclude, good to fair and significant correlations between $\log \left(A c t_{\max }\right)$ and $\log \left(P_{\mathrm{EH}}\right)$ of the experimental data set $\left(R^{2}=0.50, p=4.6^{*} 10^{-23}\right)$ and the flexibility data set $\left(R^{2}=0.22, p=\right.$ $0.6^{*} 10^{-2}$ ) were found. Hence, promiscuous EHs have higher maximum specific activities.

\subsection{Specific EHs prefer to hydrolyze large and flexible esters}

Next, we investigated, which of the 96 esters was preferentially hydrolyzed by EHs with different $P$ EH. As a criterion, we chose the number of freely rotatable bonds of an ester, TA (see section 2.7). We did so because TA is a combined measure for an ester's size and conformational dynamics (57). To account for the uneven distribution of esters in our data set

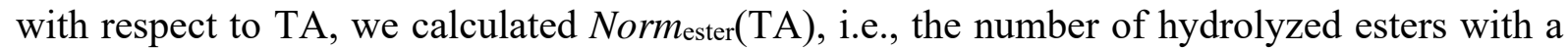
specific TA (Esterhydrolysed (TA)) divided by the total number of esters in the data set with this specific TA (Ester library(TA)) (see section 2.7) (Eq. 1). 
472 According to TA, the esters were classified into 17 groups that ranged from small esters with

473 no rotatable bond to large esters with 56 rotatable bonds (Figure 4, Table S9). Esters with three

474 (24\% of the ester library) and four (16\% of the ester library) rotatable bonds are most frequent.

475 The analysis of the experimental data set revealed that promiscuous EHs have high Normester

476 values irrespective of TA, i.e., promiscuous EHs accept a large variety of esters with different

477 sizes and degrees of conformational dynamics (Figure 4A, Table S9). In contrast, specific EHs

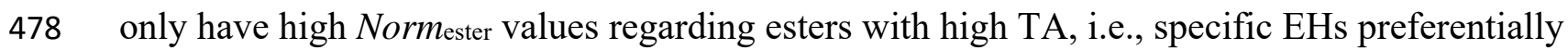

479 hydrolyze (very) large and flexible esters (Figure 4A, Table S9). The same tendency was

480 observed for the flexibility data set (Figure 4B, Table S9).

481 To conclude, promiscuous EHs accept a large variety of esters with different sizes and degrees

482 of conformational dynamics whereas specific EHs preferentially hydrolyze (very) large and 483 flexible esters. 


\section{Discussion}

The main outcomes of our analyses are I) that promiscuous EHs are significantly globally less flexible and have less flexible catalytically active residues than specific ones, II) that promiscuous EHs are significantly more thermostable, III) that promiscuous EHs have a significantly increased specific activity, and IV) that specific EHs prefer to hydrolyze large and flexible esters.

We established these relations using one of the still few experimental large-scale datasets where a diverse set of EHs was functionally assessed against a customized library of dissimilar esters (19). Functional promiscuity may arise from several conditions, including the environment of the enzyme or the concentration of a substrate, which may complicate the analysis of the molecular mechanism underlying promiscuity (5). Still, functional promiscuity ultimately is a result of recognition promiscuity (5); here, we therefore focused on substrate promiscuity (18), i.e., an enzyme carries out its typical catalytic function using non-canonical substrates, in that experimental conditions had been kept constant for the assessment of the different esterase/ester combinations (19). Almost all of the EHs were unambiguously assigned to one of the $F_{\mathrm{EH}}$ of the Arpigny and Jaeger classification, which is based mainly on a comparison of amino acid sequences (53). Except for classes with a few members only (cyclase-like EHs and the yeast family), all other classes cover at least two of the three $P_{\mathrm{EH}}$ ranges such that $P_{\mathrm{EH}}$ cannot be assigned based on the EH's class affiliation (Figure S13, Table S10) and, hence, amino acid sequence information. Even family $F_{\mathrm{IV}}$, which contains a higher proportion of substratepromiscuous EHs, also contains EHs with a small substrate range.

For scrutinizing the mechanism underlying esterase promiscuity at the atomistic level, we needed to apply comparative models of EHs, since only for $\sim 7 \%$ of the experimentally assessed EHs crystal structures were available. Restricting the generation of esterase models to sequence identities $\geq 25 \%$ with respect to available targets yielded generally good structural models both globally and locally, as also validated against cases where crystal structures are known. Throughout our study, we probed for the consistency of our analyses between subsets of EHs for which either crystal structures are known or not; we only found quantitative differences, but no qualitative ones. One of the reasons is likely that rigidity analyses were based on structural ensembles generated by multiple and $\mu$ s-long MD simulations, which has been shown to improve both global and local protein structure $(58,59)$ to the level of approaching experimental accuracy (60) and markedly increases the robustness of the results (42). We furthermore showed that results are consistent irrespective of whether EH flexibility 
characteristics were assessed globally or only for CARs, and that mobility characteristics computed directly from MD trajectories show the same trend, although the correlation with $P$ EH is insignificant. Finally, we used experimental melting temperatures of EHs as indicators for enzyme flexibility $(45,48)$, which yielded the same relation with $P_{\mathrm{EH}}$ as computed flexibility characteristics. The partial use of comparative models rather than crystal structures throughout this study may lead to concern. Yet, our consistent and robust findings indicate that when applying this workflow to novel EHs, including to those for which no crystal structure exists but a structural homolog with a sequence identity $\geq 25 \%$, it should be possible to discover enzymes with 'sufficient' substrate promiscuity to serve as a starting point for further exploration in biotechnology and synthetic organic chemistry. In that respect, the flexibility characteristics of EHs analyzed here have a notably stronger predictive power than the active site effective volume introduced earlier (19) (Figure S14, Tables S11 and S12).

The finding that promiscuous EHs are significantly globally less flexible and have less flexible catalytically active residues than specific EHs is in stark contrast to the general view of the role of structural flexibility for promiscuity $(4,5)$ : Besides the examples of CYP and $\beta$-lactamase mentioned above, the possibility of dynamically restructuring active sites has also been recognized for other systems as underlying their promiscuity (61-64). Finally, interactions between antibodies and antigens are likely the quintessential example of the canonical relationship between flexibility and binding promiscuity: As antibodies mature to become more specific, their flexibility is decreased (5).

It has been recognized that conformational changes may not always be necessary for promiscuity if a variety of substrates can be bound by partial recognition or the presence of multiple binding sites (5). However, these cases do not seem to be relevant reasons for EH promiscuity because partial recognition often is associated with catalytic inefficiency (1), which is contrary to our observation that $P_{\mathrm{EH}}$ correlates with $\mathrm{EH}$ activity, and the presence of multiple binding sites that could give rise to promiscuity is controverted by the finding that promiscuous EHs have large active site effective volumes (19), i.e., large pockets with few subpockets. Inversely, our findings of rigid promiscuous EHs may be consistent with the idea that multiple ligands can be accommodated in a single site by exploiting diverse interacting residues (Figure 5).

Our results as to specific but flexible EHs may be reconciled with a model according to which conformational changes may have been selected in EH evolution for their ability to enhance specificity in recognition (Figure 5), resulting in what has been termed conformational 
551 proofreading (16). In the case of specific EHs, flexibility may help to overcome a structural

552 mismatch between the enzyme and its substrate existing when both are in their ground states,

553 that way enhancing recognition specificity. This view is corroborated by our finding that

554 specific EHs prefer to hydrolyze large and flexible substrates: Larger substrates can form more 555 interactions with the enzyme, that way helping to overcome the deformation energy required 556 by the enzyme to optimizing the correct binding probability over the incorrect one; flexible 557 substrates can tolerate higher strains and thus can be expected to participate in more binding 558 events $(65,66)$ (Figure 5). In that respect, the relation between structural flexibility of EHs and 559 promiscuity found here is more causative than that between active site effective volume and 560 promiscuity (19), because small active site effective volumes found for specific EHs cannot 561 rationalize why specific EHs prefer to hydrolyze large and flexible substrates.

562 In summary, the combined large-scale analysis of experimental EH promiscuity and computed 563 EH flexibility reveals that promiscuous EHs are significantly less flexible than specific ones.

564 This result is counterintuitive at first but may be reconciled with a model according to which 565 multiple ligands can be accommodated in a single active site of promiscuous EHs by exploiting 566 diverse interacting residues, whereas structural flexibility in the case of specific EHs serves for 567 conformational proofreading. Our results furthermore signify that EH sequence space, charted, 568 e.g., by (meta)genomics studies, can be screened by rigidity analyses for promiscuous EHs that 569 may serve as starting points for further exploration in biotechnology and synthetic organic chemistry. 
572

573

574

575

576

577

578

579

580

581

582

583

584

585

586

587

588

589

590

591

592

593

594

595

596

597

598

599

600

601

\section{Acknowledgements}

$\mathrm{CN}$ is funded through a grant ("Vernetzungsdoktorand") provided by the Forschungszentrum Jülich. Parts of the study were supported by the German Federal Ministry of Education and Research (BMBF) through funding number 031B0837A "LipoBiocat" to HG and KEJ and through funding number 031L0182 "InCelluloProtStruct" to HG, the German Research Foundation (DFG) through funding no. INST 208/704-1 FUGG to HG and INST 208/654-1 FUGG to KEJ, as well as the state of North Rhine Westphalia (NRW) and the European Regional Development Fund (EFRE) through funding no. 34-EFRE-0300096 "CLIBKompetenzzentrum Biotechnologie (CKB)" to HG. HG is grateful for computational support and infrastructure provided by the "Zentrum für Informations- und Medientechnologie" (ZIM) at the Heinrich Heine University Düsseldorf. HG gratefully acknowledges the computing time granted by the John von Neumann Institute for Computing (NIC) and provided on the supercomputer JUWELS at Jülich Supercomputing Centre (JSC) (user IDs: HKF7; protil (project ID: 15956)) (67). HG is grateful to OpenEye for an academic license. MF acknowledges the grant 'INMARE' from the European Union's Horizon 2020 (grant agreement no. 634486) and BIO2017-85522-R from the Ministerio de Ciencia, Innovación y Universidades (MCIU), Agencia Estatal de Investigación (AEI), Fondo Europeo de Desarrollo Regional (FEDER) and European Union (EU). CC thanks the Ministerio de Economía y Competitividad and FEDER for a PhD fellowship (Grant BES-2015-073829). The authors are grateful to David Almendral and Ruth Matesanz for their support of CD analysis.

\section{Authors contributions}

HG and KEJ conceived the study. CN analyzed the experimental data, performed structure prediction, MD simulations and CNA computations, analyzed the computational data, and wrote the manuscript together with HG. DM initially contributed to the structure prediction. BD performed similarity analysis of esters. MF and CC measured and analyzed melting temperatures and determined kinetic parameters. HG supervised and managed the project. All authors reviewed and approved the manuscript.

\section{Conflict of interest}

The authors declare no financial and non-financial competing interests. 


\section{Figures}

A

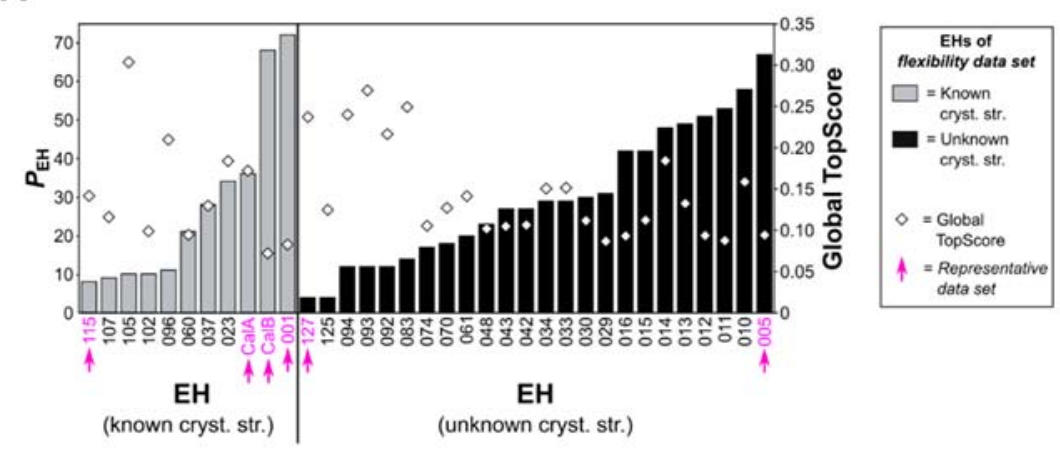

B
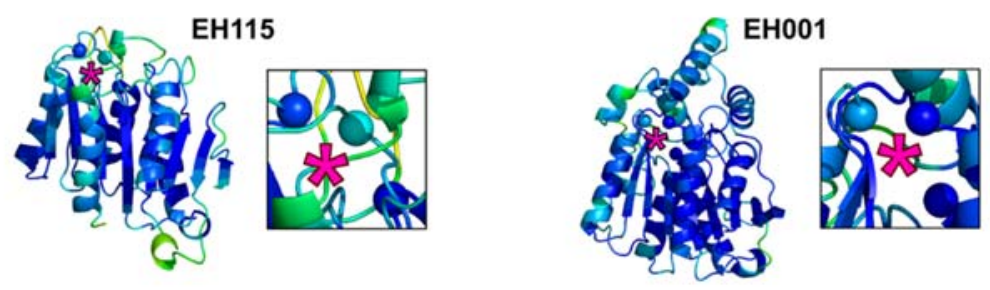

C
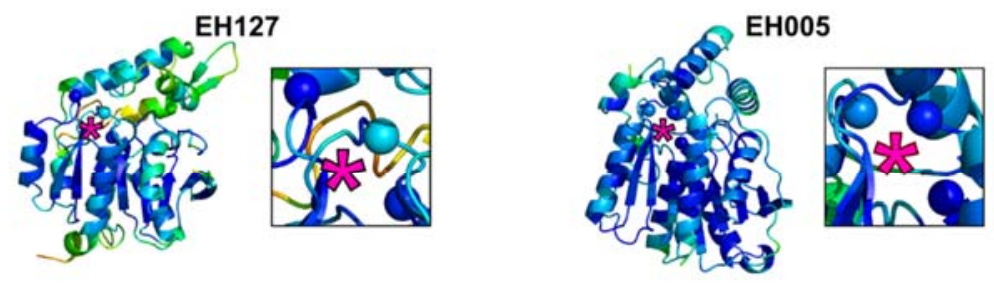

D
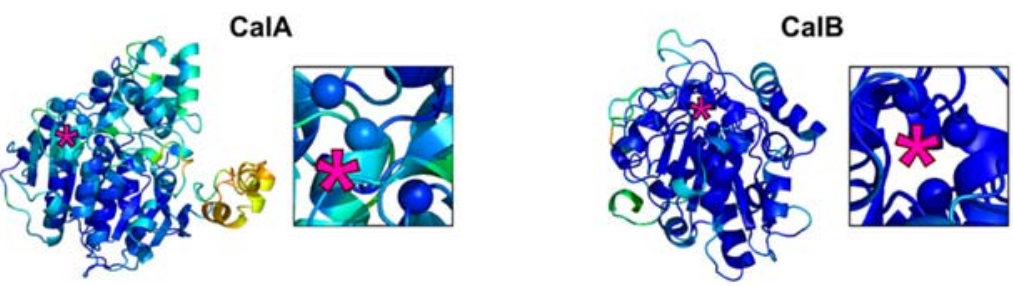

\begin{tabular}{ll}
\multicolumn{2}{c}{ Residue-wise TopScore } \\
\hline 0.1 & \\
\hline &
\end{tabular}

Figure 1: Comparative modeling of EHs. (A) Based on sequence data provided by a largescale study from Martínez-Martínez et al. (19), comparative models were generated for $35 \mathrm{EHs}$ with known (left, $11 \mathrm{EHs)}$ ) and unknown (right, $24 \mathrm{EHs)} \mathrm{crystal} \mathrm{structures} \mathrm{using} \mathrm{TopModel} \mathrm{(26).}$ These EHs constitute the flexibility data set. The EHs vary in $P_{\mathrm{EH}}$ (left ordinate, bars) and global TopScores (right ordinate, diamonds). Six EHs were selected as representatives of the flexibility data set (termed representative data set) as indicated by magenta arrows. The quality of the comparative models of (B) EHs with known crystal structures and lowest (EH115) or highest $P_{\mathrm{EH}}$ (EH001), (C) EHs with unknown crystal structures and lowest (EH127) or highest $P_{\mathrm{EH}}$ (EH005), and (D) commercial EHs with highest (CalA) or lowest $P_{\mathrm{EH}}(\mathrm{CalB})$ was evaluated by TopScore (31). For each comparative model the residue-wise TopScore is shown: A good (bad) homology model shows a low (high) residue-wise TopScore (see color scale at the bottom). Insets depict CARs (spheres) within an EH. For clarity the position of CARs is indicated by magenta stars. 


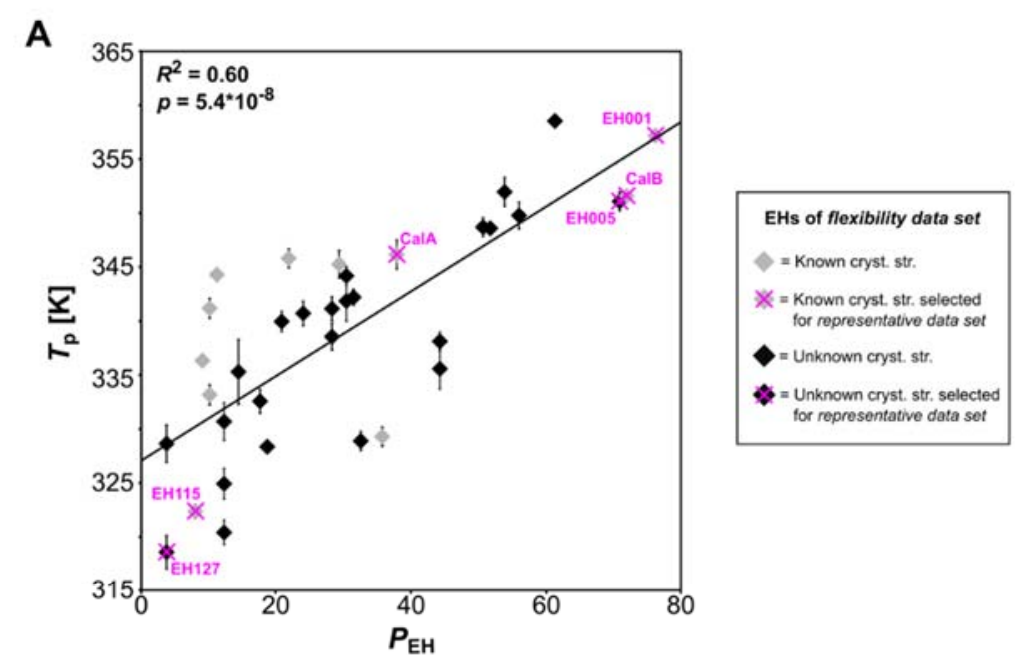

B
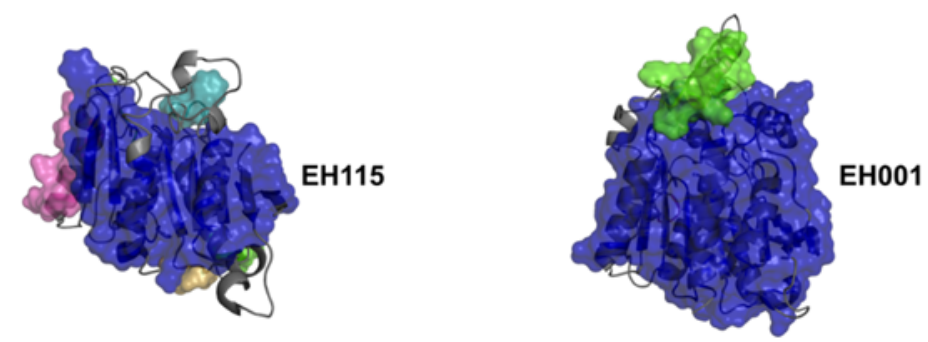

C
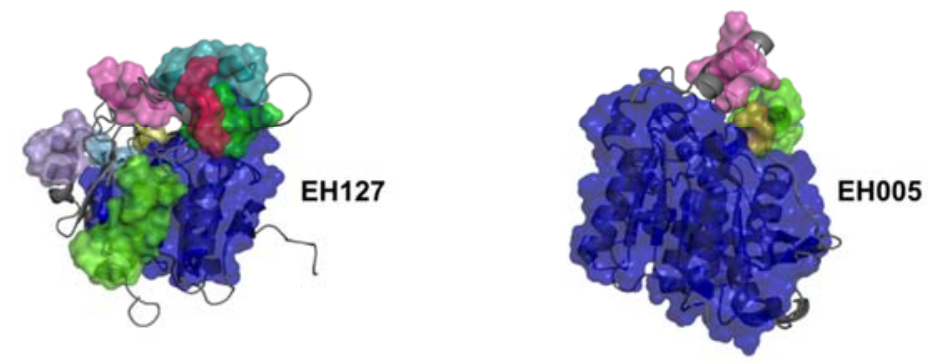

D
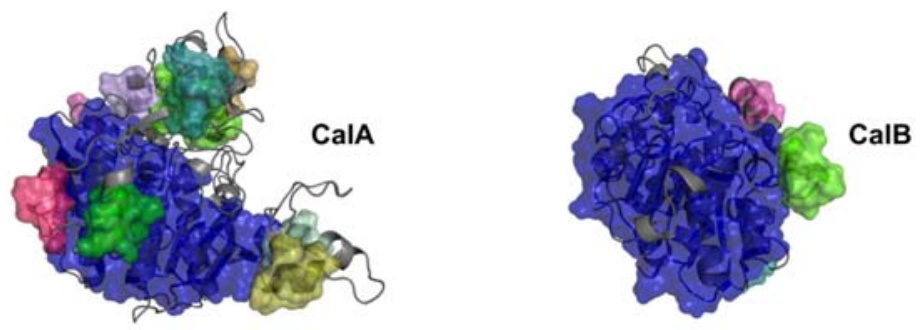

Figure 2: Correlation of $\boldsymbol{T}_{\mathbf{p}}$ versus $\boldsymbol{P}_{\mathrm{EH}}$ (A) Correlation between predicted $T_{\mathrm{p}}$ based on the global index $H_{\text {type } 2}$ and $P_{\mathrm{EH}}$ for the flexibility data set. Data points colored grey (black) represent comparative models of EHs with (un)known crystal structures. The representative data set is indicated by magenta crosses. Error bars show the SEM over five independent MD simulations of $1 \mu$ s length each. Rigid cluster decomposition at $332 \mathrm{~K}$ during the thermal unfolding simulation of (B) EHs with known crystal structures and lowest (EH115) or highest $P_{\mathrm{EH}}$ (EH001), (C) EHs with unknown crystal structures and lowest (EH127) or highest $P_{\mathrm{EH}}$ (EH005), and (D) commercial EHs with lowest (CalA) or highest $P_{\mathrm{EH}}(\mathrm{CalB})$. Rigid clusters are represented as uniformly colored blue, green, pink, cyan, and magenta bodies in the descending order of their sizes. 

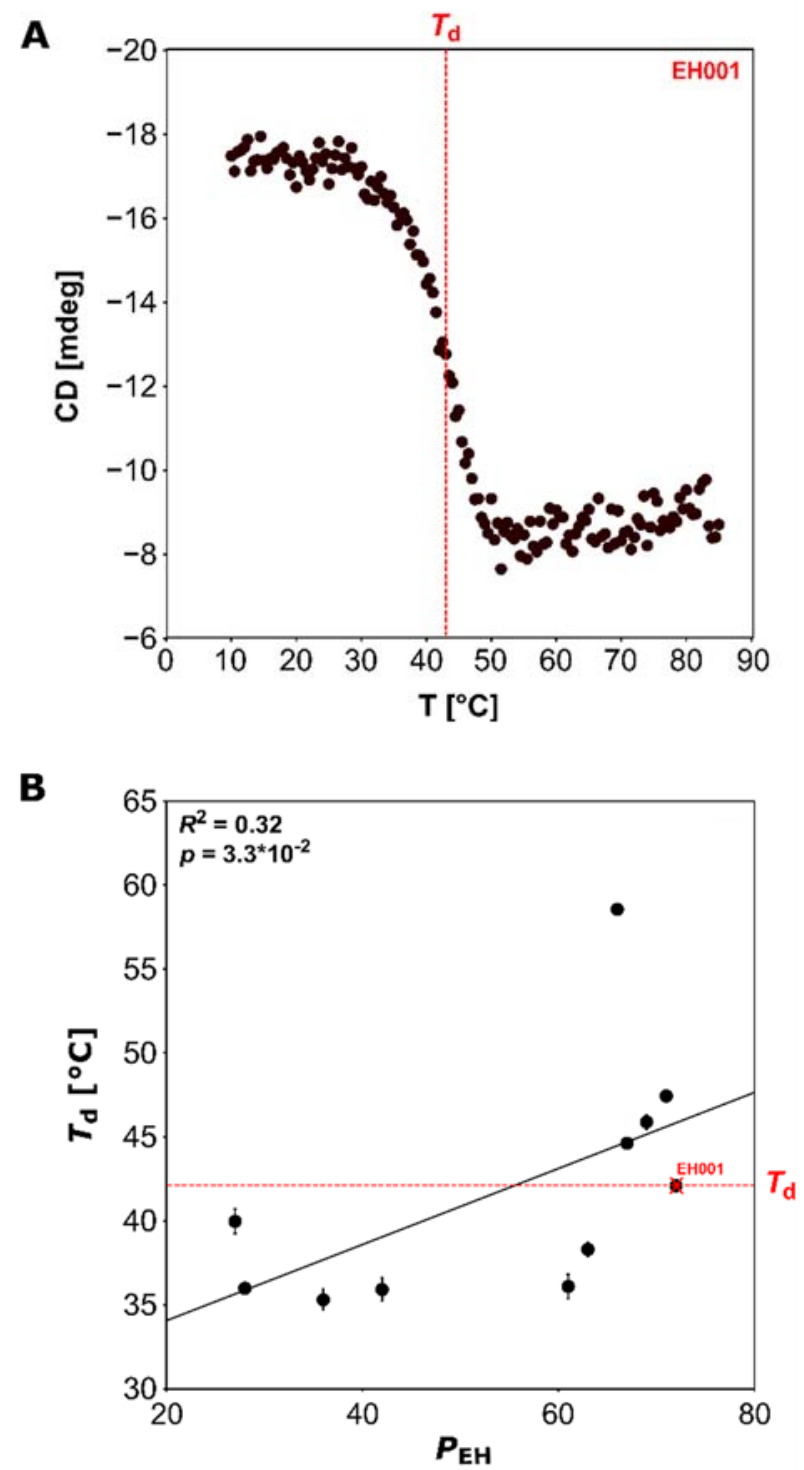

Figure 3: Determination of $\boldsymbol{T}_{\mathbf{d}}$ via CD spectroscopy. (A) Exemplary CD spectrum of EH001. The ellipticity changes in mdeg at $220 \mathrm{~nm}$ was plotted against the temperature, resulting in a sigmoidal curve. The inflection point was used to obtain the $T_{\mathrm{d}}$ value (dotted line). (B) Correlation between $T_{\mathrm{d}}$ and $P_{\mathrm{EH}}$ for $12 \mathrm{EHs}$ of FIV. 
A

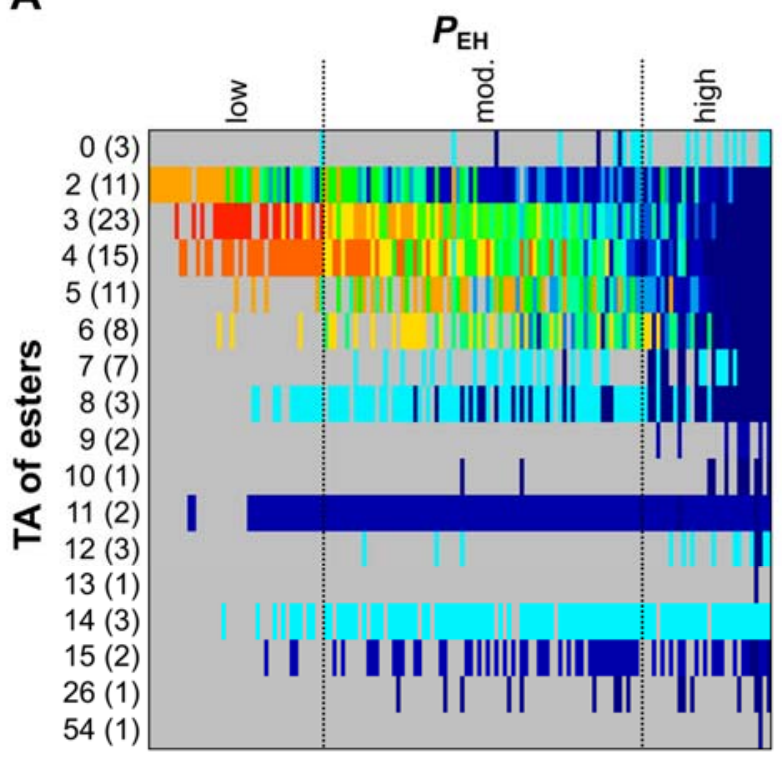

EH

B

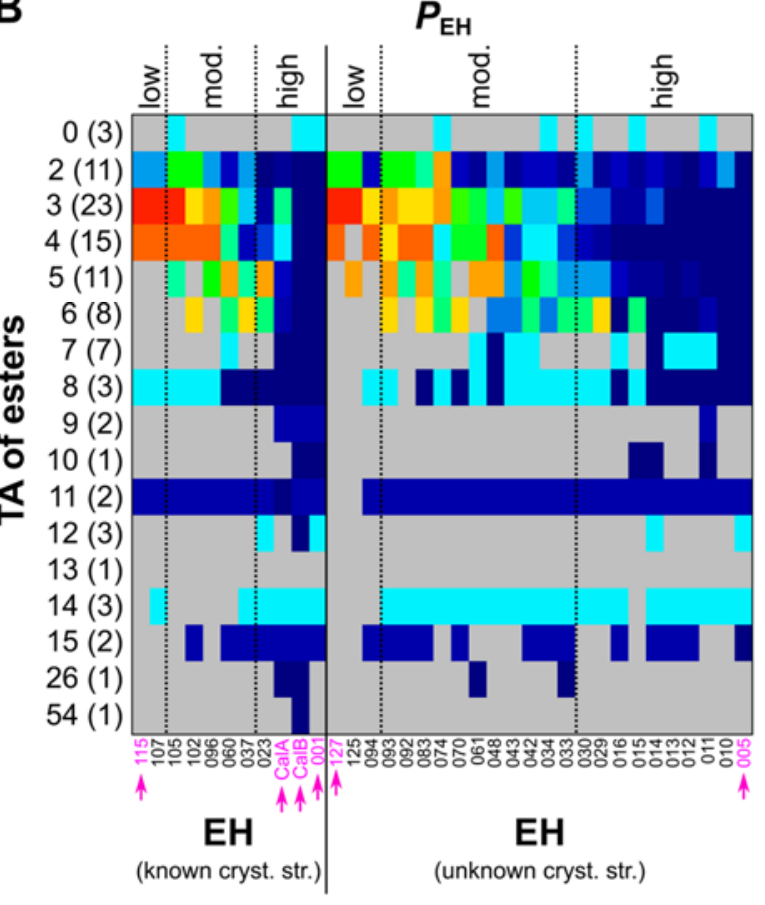

Normalized proportion of hydrolyzed esters
with a specific TA [\%]

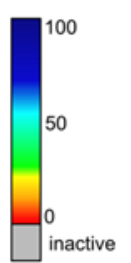

Figure 4: Relation between the number of esters' TA and $\boldsymbol{P}_{\mathrm{EH}}$. Relation between Ester ${ }_{\text {norm, }}$ i.e., the relative proportion of the number of hydrolyzed esters with a specific TA, and $P_{\mathrm{EH}}$ of (A) the experimental data set and (B) the flexibility data set containing EHs with known crystal structures (left), EHs with unknown crystal structures (right), and EHs constituting the representative data set (indicated by magenta arrows). TA was calculated based on SMILES codes of 96 esters provided by Martínez-Martínez et al. (19). A blue (red) color indicates that the EH hydrolyzes many (few) esters with a specific TA relative to the total number of esters in the data set with this specific TA (see color scale on the right); the total number of esters with a specific TA is given in brackets on the y-axis. $P_{\mathrm{EH}}$ is defined as low if the EH hydrolyzes $\leq 9$ esters, as moderate if the EH hydrolyzes between 10 and 29 esters, and as high if the EH hydrolyzes $\geq 30$ esters. 

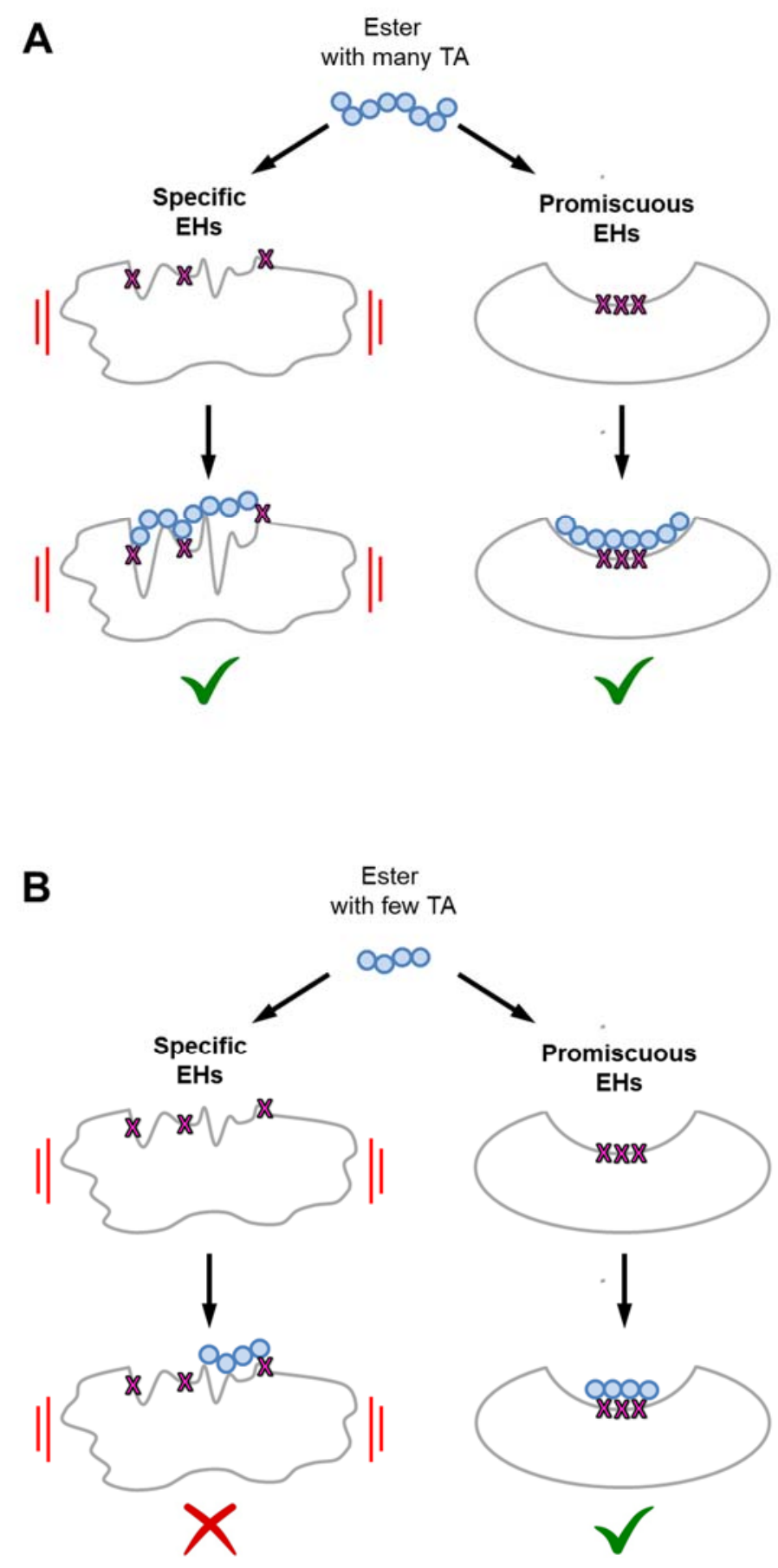

Figure 5: Mechanistic model of EH flexibility, ligand size and conformational dynamics affecting $\boldsymbol{P}_{\mathbf{E H}}$. Impact of esters with (A) many or (B) few TA on specific, and hence more flexible (left), and promiscuous, and hence more rigid (right), EHs. Ligand parts connected by TA are represented as blue circles. Specific EHs and large ligands with many TA can mutually adapt (panel A, left), and promiscuous EH can bind large ligands (panel A, right) and small ligands (panel $\mathbf{B}$, right) exploiting different interaction partners. Small (and/or rigid) ligands are not able to lead to a structural adaptation of specific EHs (panel $\mathbf{B}$, left), though, resulting in conformational proofreading. The red bars indicate the flexibility of the EHs. A green tick (red cross) indicates that ester cleavage is (not) catalyzed. 


\section{References}

1. Copley SD (2017) Shining a light on enzyme promiscuity. Curr. Opin. Struct. Biol. 47:167-175.

2. Chen R, et al. (2017) Molecular insights into the enzyme promiscuity of an aromatic prenyltransferase. Nat. Chem. Biol. 13(2):226-234.

3. Huang $\mathrm{H}$, et al. (2015) Panoramic view of a superfamily of phosphatases through substrate profiling. Proc. Natl. Acad. Sci. U.S.A. 112(16):E1974-E1983.

4. Khersonsky O \& Tawfik DS (2010) Enzyme promiscuity: a mechanistic and evolutionary perspective. Annu. Rev. Biochem. 79:471-505.

5. Nobeli I, Favia AD, \& Thornton JM (2009) Protein promiscuity and its implications for biotechnology. Nat. Biotechnol. 27(2):157-167.

6. Eisenmesser EZ, Bosco DA, Akke M, \& Kern D (2002) Enzyme dynamics during catalysis. Science 295(5559):1520-1523.

7. Henzler-Wildman KA, et al. (2007) A hierarchy of timescales in protein dynamics is linked to enzyme catalysis. Nature 450(7171):913-916.

8. Holliday MJ, Camilloni C, Armstrong GS, Vendruscolo M, \& Eisenmesser EZ (2017) Networks of dynamic allostery regulate enzyme function. Structure 25(2):276-286.

9. Goodey NM \& Benkovic SJ (2008) Allosteric regulation and catalysis emerge via a common route. Nat. Chem. Biol. 4(8):474-482.

10. Saavedra HG, Wrabl JO, Anderson JA, Li J, \& Hilser VJ (2018) Dynamic allostery can drive cold adaptation in enzymes. Nature 558(7709):324-328.

11. Tzeng SR \& Kalodimos CG (2011) Protein dynamics and allostery: an NMR view. Curr. Opin. Struct. Biol. 21(1):62-67.

12. Skopalík J, Anzenbacher P, \& Otyepka M (2008) Flexibility of human cytochromes P450: molecular dynamics reveals differences between CYPs 3A4, 2C9, and 2A6, which correlate with their substrate preferences. J. Phys. Chem. B. 112(27):8165-8173.

13. Hendrychová T, et al. (2011) Flexibility of human cytochrome P450 enzymes: molecular dynamics and spectroscopy reveal important function-related variations. Biochim. Biophys. Acta. 1814(1):58-68.

14. Ekroos M \& Sjögren T (2006) Structural basis for ligand promiscuity in cytochrome P450 3A4. Proc. Natl. Acad. Sci. U.S.A. 103(37):13682-13687.

15. Zou T, Risso VA, Gavira JA, Sanchez-Ruiz JM, \& Ozkan SB (2015) Evolution of conformational dynamics determines the conversion of a promiscuous generalist into a specialist enzyme. Mol. Biol. Evol. 32(1):132-143.

16. Savir Y \& Tlusty T (2007) Conformational proofreading: the impact of conformational changes on the specificity of molecular recognition. PloS One 2(5):e468.

17. Ferrer M, et al. (2016) Estimating the success of enzyme bioprospecting through metagenomics: current status and future trends. Microb. Biotechnol. 9(1):22-34.

18. Hult K \& Berglund P (2007) Enzyme promiscuity: mechanism and applications. Trends. Biotechnol. 25(5):231-238.

19. Martinez-Martinez M, et al. (2017) Determinants and prediction of esterase substrate promiscuity patterns. ACS Chem. Biol. 13(1):225-234.

20. Radestock S \& Gohlke H (2008) Exploiting the link between protein rigidity and thermostability for data-driven protein engineering. Eng. Life Sci. 8(5):507-522.

21. Pfleger C, Rathi PC, Klein DL, Radestock S, \& Gohlke H (2013) Constraint Network Analysis (CNA): a Python software package for efficiently linking biomacromolecular structure, flexibility,(thermo-) stability, and function. J. Chem. Inf. Model. 53:10071015. 
22. Krüger DM, Rathi PC, Pfleger C, \& Gohlke H (2013) CNA web server: rigidity theorybased thermal unfolding simulations of proteins for linking structure,(thermo-) stability, and function. Nucleic Acids Res. 41(W1):W340-W348.

23. Hermans SM, Pfleger C, Nutschel C, Hanke CA, \& Gohlke H (2017) Rigidity theory for biomolecules: concepts, software, and applications. Comput. Mol. Sci. 7(4):e1311.

24. de Godoy Daiha K, Angeli R, de Oliveira SD, \& Almeida RV (2015) Are lipases still important biocatalysts? A study of scientific publications and patents for technological forecasting. PloS One 10(6): $\mathrm{e} 0131624$.

25. Ferrer M, et al. (2015) Biodiversity for biocatalysis: a review of the $\alpha / \beta$-hydrolase fold superfamily of esterases-lipases discovered in metagenomes. Biocatal. Biotranfor. 33(5-6):235-249.

26. Mulnaes D, et al. (2020) TopModel: Template-based protein structure prediction at low sequence identity using top-down consensus and deep neural networks. J. Chem. Theory. Comput. 16:1953-1967.

27. Gohlke H, et al. (2013) Binding region of alanopine dehydrogenase predicted by unbiased molecular dynamics simulations of ligand diffusion. J. Chem. Inf. Model. 53(10):2493-2498.

28. Widderich N, et al. (2014) Molecular dynamics simulations and structure-guided mutagenesis provide insight into the architecture of the catalytic core of the ectoine hydroxylase. J. Mol. Biol. 426(3):586-600.

29. Zhang Z, et al. (2016) Determinants of FIV and HIV Vif sensitivity of feline APOBEC3 restriction factors. Retrovirology 13(1):46.

30. Milić D, et al. (2018) Recognition motif and mechanism of ripening inhibitory peptides in plant hormone receptor ETR1. Sci. Rep. 8(1):3890.

31. Mulnaes D \& Gohlke H (2018) TopScore: Using Deep Neural Networks and Large Diverse Data Sets for Accurate Protein Model Quality Assessment. J. Chem. Theory Comput. 14(11):6117-6126.

32. Chovancova E, et al. (2012) CAVER 3.0: a tool for the analysis of transport pathways in dynamic protein structures. PLoS Comput. Biol. 8(10):e1002708.

33. Salomon-Ferrer R, Götz AW, Poole D, Le Grand S, \& Walker RC (2013) Routine microsecond molecular dynamics simulations with AMBER on GPUs. 2. Explicit solvent particle mesh Ewald. J. Chem. Theory Comput. 9(9):3878-3888.

34. Case DA, et al. (2019) AMBER 2019University of California, San Francisco).

35. Hopkins CW, Le Grand S, Walker RC, \& Roitberg AE (2015) Long-time-step molecular dynamics through hydrogen mass repartitioning. J. Chem. Theory Comput. 11(4):1864-1874.

36. Jacobs DJ, Rader AJ, Kuhn LA, \& Thorpe MF (2001) Protein flexibility predictions using graph theory. Proteins 44(2):150-165.

37. Hespenheide B, Jacobs D, \& Thorpe M (2004) Structural rigidity in the capsid assembly of cowpea chlorotic mottle virus. J. Condens. Matter Phys. 16(44):S5055.

38. Jacobs DJ \& Thorpe MF (1995) Generic rigidity percolation: the pebble game. Phys. Rev. Lett. 75(22):4051.

39. Rader AJ, Hespenheide BM, Kuhn LA, \& Thorpe MF (2002) Protein unfolding: rigidity lost. Proc. Natl. Acad. Sci. U.S.A. 99(6):3540-3545.

40. Livesay DR \& Jacobs DJ (2006) Conserved quantitative stability/flexibility relationships (QSFR) in an orthologous RNase H pair. Proteins 62(1):130-143.

41. Radestock S \& Gohlke H (2011) Protein rigidity and thermophilic adaptation. Proteins 79(4):1089-1108.

42. Pfleger C \& Gohlke H (2013) Efficient and robust analysis of biomacromolecular flexibility using ensembles of network topologies based on fuzzy noncovalent constraints. Structure 21(10):1725-1734. 
43. Pfleger C, Radestock S, Schmidt E, \& Gohlke H (2013) Global and local indices for characterizing biomolecular flexibility and rigidity. J. Comput. Chem. 34(3):220-233.

44. Rathi PC, Radestock S, \& Gohlke H (2012) Thermostabilizing mutations preferentially occur at structural weak spots with a high mutation ratio. J. Biotechnol. 159(3):135-144.

45. Rathi PC, Jaeger K-E, \& Gohlke H (2015) Structural rigidity and protein thermostability in variants of lipase A from Bacillus subtilis. PloS One 10(7):e0130289.

46. Dick M, et al. (2016) Trading off stability against activity in extremophilic aldolases. Sci. Rep. 6:17908.

47. Rathi PC, Fulton A, Jaeger K-E, \& Gohlke H (2016) Application of rigidity theory to the thermostabilization of lipase A from Bacillus subtilis. PLoS Comput. Biol. 12(3):e1004754.

48. Nutschel C, et al. (2020) Systematically scrutinizing the impact of substitution sites on thermostability and detergent tolerance for Bacillus subtilis lipase A. J. Chem. Inf. Model. 60:1568-1584.

49. Gohlke H, Kuhn LA, \& Case DA (2004) Change in protein flexibility upon complex formation: analysis of Ras-Raf using molecular dynamics and a molecular framework approach. Proteins 56(2):322-337.

50. Eisenthal R, Danson MJ, \& Hough DW (2007) Catalytic efficiency and kcat/KM: a useful comparator? Trends Biotechnol. 25(6):247-249.

51. Nath A \& Atkins WM (2008) A quantitative index of substrate promiscuity. Biochemistry 47(1):157-166.

52. Hawkins PC, Skillman AG, \& Nicholls A (2007) Comparison of shape-matching and docking as virtual screening tools. J. Med. Chem. 50(1):74-82.

53. Arpigny JL \& Jaeger K-E (1999) Bacterial lipolytic enzymes: classification and properties. Biochem. J. 343(1):177-183.

54. Greenfield NJ (2006) Using circular dichroism collected as a function of temperature to determine the thermodynamics of protein unfolding and binding interactions. Nat. Protoc. 1(6):2527-2535.

55. Mayr H \& Ofial AR (2006) The reactivity-selectivity principle: an imperishable myth in organic chemistry. Angew. Chem. Int. Ed. Engl. 45(12):1844-1854.

56. Wells PR (1963) Linear Free Energy Relationships. Chem. Rev. 63(2):171-219.

57. Böhm H-J (1994) The development of a simple empirical scoring function to estimate the binding constant for a protein-ligand complex of known three-dimensional structure. J. Comput. Aided Mol. Des. 8(3):243-256.

58. Feig M (2017) Computational protein structure refinement: Almost there, yet still so far to go. Wiley Interdiscip Rev Comput Mol Sci 7(3).

59. Heo L \& Feig M (2018) Experimental accuracy in protein structure refinement via molecular dynamics simulations. Proc. Natl. Acad. Sci. U. S. A. 115(52):13276-13281.

60. Heo L, Arbour CF, \& Feig M (2019) Driven to near-experimental accuracy by refinement via molecular dynamics simulations. Proteins 87(12):1263-1275.

61. Seibert CM \& Raushel FM (2005) Structural and catalytic diversity within the amidohydrolase superfamily. Biochemistry 44(17):6383-6391.

62. Oppermann U, et al. (2003) Short-chain dehydrogenases/reductases (SDR): the 2002 update. Chem. Biol. Interact. 143-144:247-253.

63. Fushinobu S, Nishimasu H, Hattori D, Song H-J, \& Wakagi T (2011) Structural basis for the bifunctionality of fructose-1, 6-bisphosphate aldolase/phosphatase. Nature 478(7370):538-541.

64. Du J, Say RF, Lü W, Fuchs G, \& Einsle O (2011) Active-site remodelling in the bifunctional fructose-1, 6-bisphosphate aldolase/phosphatase. Nature 478(7370):534537. 
65. Stockwell GR \& Thornton JM (2006) Conformational diversity of ligands bound to proteins. J. Mol. Biol. 356(4):928-944.

66. Perola E \& Charifson PS (2004) Conformational analysis of drug-like molecules bound to proteins: an extensive study of ligand reorganization upon binding. J. Med. Chem. 47(10):2499-2510.

67. Krause D (2019) JUWELS: Modular Tier-0/1 Supercomputer at the Jülich Supercomputing Centre. JLSRF 5:A135. 